\title{
Peculiaridades de la lectura táctil del braille. Un estudio empírico
}

\author{
AlBERTO ROSA y JUAN ANTONIO HUERTAS \\ Universidad Autónoma de Madrid
}

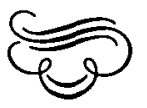

\section{Resumen}

Este articulo ofrece una discusión sobre las posibles causas que pueden explicar por qué el desamollo cognitivo de los ciegos presenta retrasos importantes en el periodo de las operaciones concretas, mientras que no presenta diferencias respecto a los videntes en tareas de tipo proposicional. Para ello se examinan tres posibles explicaciones: la influencia del modo de escolarización, las caracteristicas del tacto y la remediación verbal. Se acaba concluyendo que estas dos últimas hipótesis son las que ofrecen una explicación más convincente. Los datos que aquî se aportan parećen sostener la bipótesis dual sobre la representación de la información en la memoria.

Palabras clave: Ceguera, Lectura braille.

\section{An empirical study of braille reading}

\section{Abstract}

This paper presents results of a research on braille reading with visually impaired people of different age and reading skill. Data on three aspects are reported here: reading speed, patterms of hand movements and types of reading mistakes. Results show that braille tactile reading is much slower than visual print reading at any level of reading bability. It seems to be pattems of hand movements which correspond to different grades of reading skill. And there are some reading errors typical of braille reading which can be claimed to be a consequence of the peculiarities of the tactual system and the characteristics of the braille alphabet.

Key words: Blind, Braille reading.

Direcciōn de los autores: Facultad de Psicología. Universidad Autónoma de Madrid. Cantoblanco. 28049 Madrid.

Agradecimientos: Este trabajo ha podido realizarse gracias a una ayuda de investigación educativa del C.I.D.E. tramitada a través del I.C.E. de la Universidad Autónoma de Madrid. En la administración de pruebas y parte del tratamiento de los datos participó Cristina Martínez de Aragón Esquivias. 


\section{INTRODUCCION}

La lectura braille presenta un conjunto de características específicas a las que enseguida nos referiremos, y que la hacen de gran interés de cara a un estudio profundo de los procesos de lectura. Esto, junto al propio interés intrínseco que este tema tiene de cara a la educación de los deficientes visuales, es el motivo que nos ha guiado a emprender un estudio cuyos resultados aquí presentamos. Por otra parte, nuestro trabajo pretende llenar un hueco dentro de la investigación sobre la ceguera en el ámbito lingüístico español, puesto que, por lo que sabemos, no existen trabajos experimentales en nuestro idioma que aborden con cierta profundidad un estudio de la lecto-escritura braille en lengua castellana.

Como es sabido el sistema de escritura braille está diseñado para su uso a través de la modalidad táctil, sirviéndose para ello de puntos que aparecen en relieve sobre una hoja de cartulina o de plástico. La unidad básica del sistema braille está constituida por la «celdillas dentro de cual se sitúan un conjunto de puntos en seis posibles posiciones, cada una de ellas designada por un número específico (ver figura 1).

Por lo que se refiere a las características psicofísicas del braille parece que la disposición de los puntos, tamaño de las celdillas, distancia entre líneas, etc., parecen estar próximas a una situación óptima para un uso intensivo (Uniform Type Comittee, 1913; Latimer, 1920; Meyers y Ethington, 1956; Calving y Clark, 1958).

Entre los problemas que el braille presenta hay dos especialmente destacables: el volumen de los textos que en él se escriben (una página escrita en tinta puede llegar a ocupar hasta nueve escritas en braille) y la lenta velocidad de su lectura. Para tratar de obviarlos existe un sistema de abreviaturas que ahorra espacio. Este sistema es conocido como Braille tipo 2 en los países anglosajones y como estenografía en España. Estos sistemas comprimidos presentan la desventaja de que la misma configuración de puntos puede tener significados diferentes en función del contexto.

La lectura se realiza deslizando las yemas de los dedos de izquierda a derecha a lo largo de las líneas que constituyen el texto. La escritura puede realizarse de dos maneras, de forma manual, o utilizando una máquina de escribir «Perkins». La peculiaridad de cada una de ellas merece un comentario aparte.

La escritura manual se realiza introduciendo la hoja de papel en un bastidor cuyos lados verticales presentan unos orificios en los cuales se inserta la «pautas. Este instrumento consiste en una regleta en la que aparecen perforados unas líneas de orificios rectangulares que se corresponden con las aceldillas». El sujeto escribe presionando con un punzón en las posiciones correspondientes de cada celdilla que identifican el signo que se pretende escribir. Este procedimiento exige que la escritura se realice de derecha a izquierda, y que cada signo deba escribirse rotando 180 grados respecto a su posición cuando se lee. Es decir, los signos aparecen «en espejo» en la lectura respecto a su posición en la escritura.

La máquina Perkins es una mắquina de escribir que dispone de una tecla para cada uno de los seis puntos que constituyen cada «células y de otra que actúa como espaciador. Los puntos aparecen de abajo hacia arriba en el papel sobre el que se escribe, pero el sujeto para poder palparlos precisa desplazar su mano desde el teclado hacia la parte posterior del «carro» de la máquina. 
Cada célula se escribe presionando simultáneamente las teclas correspondientes al signo que se quiere escribir.

La existencia de este alfabeto no implica que de una forma automática los ciegos tengan un acceso a la lecto-escritura en todo similar al de los videntes, sino que tanto la propia estructura del braille como la modalidad sensorial háptica sobre la que se sustenta hacen que los procesos de lecto-escritura de los invidentes tenga algunas peculiaridades. Las páginas que siguen pretenden precisamente explorarlas.

\section{Movimientos de manos en la lectura háptica del braille}

De la revisión de la literatura se desprende claramente que los sujetos al leer utilizan únicamente los dedos índices, sin que en ningún trabajo se reseñe que en situaciones de lectura libre los deficientes visuales usen espontáneamente ningún otro dedo.

Por lo que se refiere a los movimientos de manos en la lectura parece darse un continuo en el que los sujetos empiezan leyendo con una sola mano para, progresivamente, ir incorporando la segunda, primero con una función auxiliar y, luego, en el caso de lectores muy expertos, leyendo con las dos manos al mismo tiempo.

La cuestión del papel de cada mano tiene una larga historia. Ya Heller (1904), un alumno de Wundt, se ocupó de este tema diferenciando entre una función sintética para una mano y analítica para otra. Kusajima (1974) rechaza la interpretación de Heller y sostiene que sólo es una mano la que realmente lee, quedando la otra en una función auxiliar, aunque la cooperación entre las dos permite mejorar la velocidad de lectura.

Según Kusajima, las funciones que realiza el dedo auxiliar son: seguir al dedo lector aumentando el campo perceptivo y reduciendo así la posibilidad de pasarse inadvertidamente una línea; ayudar a precisar la constante localización del dedo lector; y, sobre todo, prestar un papel auxiliar de primera magnitud en los movimientos de cambio de línea, localizando el principio de la línea siguiente y ahorrando tiempo.

Por lo que se refiere a los patrones de movimiento de la lectura con dos manos, este autor informa de cuatro tipos de «adaptaciones» graduadas en función de la destreza lectora (su muestra incluía sujetos entre 10 y 23 años de edad):

1. Sólo lee un dedo, el otro espera al inicio de la siguiente línea donde ambos se juntan de nuevo.

2. Ambos dedos se mueven uno junto al otro tocándose ligeramente y siguiendo un curso exactamente paralelo, tanto al leer como al cambiar de línea.

3. Desde el inicio de cada línea y hasta cerca del final ambos dedos se mueven juntos y tocándose ligeramente, pero cerca del final de la línea se separan. El derecho lee el resto del renglón y se asegura de que ha terminado, entonces salta de línea y se reúne con el otro que había saltado antes rápidamente. En ocasiones el dedo izquierdo ha iniciado la lectura de la segunda línea un poco antes de que el derecho haya bajado. En los casos en que el dedo lector es el izquierdo sus roles deben intercambiarse hacia el final del renglón.

4. El dedo izquierdo lee la mitad izquierda de la línea, mientras que el derecho lee la derecha. Los dos dedos se juntan en el centro, en este momento el dedo izquierdo deja al derecho que temine esta línea, mientras que el izquierdo salta al inicio de la siguiente y la lee hasta el centro, donde el dedo 
derecho se le reúne. Esto quiere decir que el lector no pierde tiempo, pues un dedo está siempre leyendo y el otro saltando de línea.

Este último tipo de exploración es similar al encontrado por Mousty, Bertelson y D'Alimonte (1985) en los sujetos que estudiaron, todos ellos ciegos adultos y lectores expertos. Según estos autores la mayoría de las líneas son exploradas según un patrón mixto que combina la exploración conjunta con la exploración disjunta. La mano izquierda lee sola un primer segmento del texto, se junta con la mano derecha y las dos leen juntas un segundo segmento, la mano izquierda se separa y va al comienzo de la línea siguiente mientras la mano derecha termina de leer la línea. De esta forma, se establecen tres trayectos distintos: trayecto unimanual izquierdo (TUI), trayecto bimanual (TB), y trayecto unimanual derecho (TUD).

Existen grandes diferencias individuales en la amplitud de los trayectos, aunque la total supresión del trayecto bimanual es extraordinariamente poco frecuente. La mano izquierda, normalmente no inicia el movimiento de cambio de línea hasta encontrarse con la derecha y hacer el TB. En algunas ocasiones se observa un TB que ocupa toda la línea, incluyendo un movimiento de cambio de renglón conjunto, si bien no es ésta una conducta que aparezca sistemáticamente.

Un fenómeno interesante, es el que estos autores denominan «exploración disjunta simultánea», en el que la mano izquierda inicia la lectura de la nueva línea mientras la derecha todavía está leyendo el renglón anterior. Este comportamiento es muy frecuente, observándose que sólo cuatro de los sujetos estudiados no presentaron este tipo de movimiento en alguna ocasión. En los demás casos el número de células leídas simultáneamente osciló entre 1 y 6 para la mano izquierda, y 1 y 8 para la derecha. Este tipo de movimiento es más normal entre los lectores rápidos que entre los lentos. La presencia de este patrón de exploración conjunta plantea el problema de la existencia de un «buffer» en donde la información recogida por cada dedo sea almacenada hasta que pueda ser integrada con la recogida simultáneamente por el otro.

\section{Velocidad de lectura}

La velocidad de la lectura háptica del braille es un tema bastante estudiado y en el que resultados obtenidos a partir de las distintas investigaciones resultan en gran parte coincidentes. Ya Hayes $(1918,1920)$ encontró una velocidad media de 30 palabras por minuto $(\mathrm{p} / \mathrm{m})$ en estudiantes de primer grado y de 83 $\mathrm{p} / \mathrm{m}$ para los de noveno, trabajos posteriores (Athearn, Campbell y Lavos, 1944; Meyers y Ethington, 1956; Nolan, 1966; Foulke, 1962) ofrecen datos prácticamente coincidentes, con los lógicos escalonamientos que cabe esperar cuando se trabaja con sujetos de diversos niveles de instrucción. Las velocidades más altas que aparecen en la literatura son las encontradas por Mousty y Bertelson (1985) con ciegos francófonos adultos y lectores expertos con velocidades medias de lectura que oscilan entre 109 y 113 p/m para textos extraídos de una novela.

En cualquier caso parece claro que el sistema braille de lectura es muy lento en comparación con la lectura visual. La explicación de esta lentitud puede encontrarse en estudios dedicados a la investigación de los factores cognitivos envueltos en la lectura táctil. Este es un tema en el que no nos vamos a detener 
por el momento, ya que el siguiente artículo de este dossier estará dedicado a examinar algunos de estos aspectos.

Existen varios estudios dedicados al posible efecto que programas de entrenamiento puedan ejercer sobre la velocidad de lectura en braille con resultados contradictorios. Mientras que los trabajos de Barraga (1963) y Kederis, Nolan y Morris (1967) no encontraron resultados positivos a sus esfuerzos para incrementar algunas habilidades de lectura. Otros estudios (Flanigan, 1964; Stockton, 1965), sin embargo, sí consiguieron resultados positivos en sus programas de entrenamiento.

Pero, quizás, el trabajo más revelador al respecto es el de Nolan y Kederis (1969). El procedimiento consistió en suministrar un programa de entrenamiento sobre reconocimiento de caracteres. Los resultados pusieron de manifiesto que el grupo experimental había mejorado en velocidad de reconocimiento de caracteres y de lectura oral, había aumentado la precisión de su identificación de caracteres y disminuido el número de sus errores en lectura oral. Sin embargo, no aparecieron diferencias significativas en la velocidad de lectura silenciosa ni en comprensión de textos leídos silenciosamente.

\section{Errores específicos en la lectura braille}

La cuestión de la legibilidad relativa de los caracteres braille y de la existencia de errores específicos producto de la disposición puntográfica de los caracteres que lo constituyen ha sido objeto, también, de trabajos de investigación.

De la revisión de la literatura se desprende que los caracteres más fácilmente legibles son los que presentan un menor número de puntos (Uniform Type Comittee, 1913), siendo especialmente difíciles los que incluyen los puntos 3 y 6, es decir, los dos correspondientes a la fila inferior (Nolan y Kederis, 1969).

Por lo que se refiere a los tipos de errores más frecuentes, estos son los de omisión y añadido de puntos (UTC, 1915; Ashcroft, 1960; Nolan y Kederis, 1969), errores a final de palabra, rotación de caracteres y sustituciones gruesas (Ashcroft, 1960; Nolan y Kederis, 1969). Es de destacar que el orden de importancia de los errores y sus magnitudes respectivas varían con el nivel escolar de los sujetos.

Un dato digno de reseñar es el encontrado por Nolan y Kederis (o.c.) de que el tiempo preciso para el reconocimiento de palabras se incrementaba conforme éstas aumentaban en longitud, disminuían en familiaridad e incluían contracciones (braille tipo 2). Es de destacar que el tiempo preciso para el reconocimiento de palabras era generalmente superior (entre un 16 y un $196 \%$ ) al preciso para reconocer cada uno de los caracteres que la componen, excepto en el caso de palabras muy familiares en el que en ocasiones, y principalmente los sujetos más diestros en la lectura, la lectura de la palabra se realizaba en tiempos de exposición demasiado breves como para que diera tiempo a su lectura completa. Este efecto se acrecentaba con la presencia de un contexto facilitador.

Estos datos parecen apoyar la interpretación de que la lectura braille se realiza letra a letra y no mediante el reconocimiento de palabras completas como en el caso de la lectura visual (Nolan y Kederis, o.c.). El efecto de la familiaridad podría, no obstante, hacer disminuir el tiempo de reconocimiento de una palabra si atendemos al fenómeno de la dominancia de las primeras letras de las palabras conocidas que señala Kusajima (1974). Esto, a su vez, permitiría explicar la existencia de errores frecuentes a finales de palabra.

Por último, de los resultados de los trabajos de Nolan (1960), Nolan y Morris 
(1960) y del suyo propio, Nolan y Kederis (1969) concluyen que el aprendizaje de la lectura táctil del braille es más lento que el de la lectura visual normal, puesto que todavía se observan progresos importantes hacia el final de la escolarización primaria. Esto es achacado por estos autores a posibles retrasos en las habilidades de discriminación táctil y auditiva, desarrollo del lenguaje y a la amplitud de experiencias de los ciegos; resumiendo todos estos factores bajo el siempre cómodo denominador común de «retrasos madurativos».

\section{OBJETIVOS DE NUESTRA INVESTIGACION}

Con nuestro trabajo pretendemos investigar los tipos de errores que aparecen en la lecto-escritura Braille en castellano, así como realizar un primer estudio del movimiento y recorrido de las manos en la lectura. Más específicamente queríamos constatar si realmente existen, y er. qué magnitud, errores en la lectura en castellano que son específicos del sistema braille, además de los comunes al proceso de lectura de videntes. Nos interesaba, también, medir la velocidad de lectura de estos sujetos ciegos, al mismo tiempo que explorar el desarrollo que se sigue en el aprendizaje de la lectura braille a través del número y del tipo de errores cometido y de la velocidad lectora, para ello utilizamos una muestra con cinco niveles de edad diferentes, desde 1 de EGB a sujetos adultos.

Por otro lado, todos los investigadores coinciden en señalar la complejidad cognitiva de los procesos de lectura. Es decir, el lector opera simultáneamente en varios niveles de procesamiento, unos más conscientes que otros. Los niveles de procesamiento más relevantes para la mayoría de los autores (cfr. De Vega, 1984) son los siguientes: a) reconocimiento de letras e integración de sílabas; b) codificación de palabras; c) codificación sintáctica; d) codificación de proposiciones; e) integración temática.

Teniendo en cuenta estos niveles de procesamiento, a la hora de hacer nuestro trabajo consideramos tres elementos fundamentales. 1. El reconocimiento háptico de las letras; 2 . La influencia del significado en palabras aisladas; 3 . La influencia de la sintaxis y del contexto.

Atendiendo a estos elementos hemos diseñado básicamente tres tipos de tareas: lectura de letras aisladas, de palabras y de textos, que serán detalladas a continuación, a partir de las cuales hemos analizado el número y tipos de errores cometidos por los sujetos. Como error hemos considerado atoda aquella lectura que el sujeto hace que no se corresponde con lo que está escrito en el texto». Hay que añadir a estas pruebas una pequeña tarea de dictado que pretendíamos que sirviese para comparar los datos de la lectura con los de la escritura.

El segundo gran objetivo del trabajo, como acabamos de decir, era el estudio de las acciones motoras que se producen al leer. En este sentido pretendemos hacer un análisis descriptivo de los movimientos de las manos, del papel de cada una de ellas, del número de dedos que intervienen en la lectura, de los movimientos que se producen en los cambios de línea y las diferencias que se dan según la edad y el grado de ceguera.

El volumen de la investigación, y la abundancia de los datos recogidos hace imposible su inclusión detallada en este artículo, cualquier persona interesada puede encontrarlos en su totalidad en Ochaita, et al (en prensa), aquí solamente incluiremos los resultados y las conclusiones más relevantes. 


\section{PROCEDIMIENTO}

\section{Diseño}

A partir de los datos de la literatura y del análisis de la tarea se esperaba que los tipos de errores que se produjesen en una o en otra magnitud fuesen: Errores de rotación (R) (cambio en la posición de la aformas de la letra girándola de izquierda a derecha, de arriba-abajo, rotándola en los dos ejes); errores de omisión o añadido (O-A) de un punto de los que configuran la letra en sí; y errores de cambio o inclusión de una letra (L). En la lista de palabras y en los textos se manipuló la variable «Efecto del Significado», con cuatro modalidades: añadir significado (A), modificar una letra para dar sentido a una palabra que no lo tenía; cambiar el significado (C) de una palabra por otra, también con sentido, al sustituir una letra por otra; mantener sin sentido a la palabra estímulo (N) y quitar significado a la palabra $(Q)$, por cambio de alguna letra/s.

\section{Pruebas Aplicadas}

Se usaron en todas las pruebas de lectura textos en braille, escritos a máquina, en el formato y tipo usualmente utilizado por los niños para la lectura táctil. Se pasaron tres grupos de tareas diferentes:

1. Lista de letras: La tarea consistía en una lista de todos los caracteres braille en castellano, tanto letras como signos de puntuación. Se incluyeron tres signos que no correspondían a ninguna letra ni signo de puntuación, se trataba de la rotación 180 grados de las letras o, s, n. Esta lista estaba escrita en una hoja de papel con una misma distancia entre los signos, que los sujetos leían en voz alta, controlándose el tiempo de lectura.

2. Lista de palabras: Se construyó una lista con 80 palabras. De ellas la mitad no tenían sentido, al habérsele incluido intencionalmente una errata en una de las letras. Las categorías de errores que se pretendian provocar fueron las siguientes:

a) Errores para dar sentido, subdivididos a su vez en tres: Errores de rotación (R-A) (en donde si se rotaba una letra se le daba sentido a la palabra v.gr. «jemonio»-«demonio»); Errores de omisión o añadido de un punto (OA-A) (de la misma forma que antes, para dar sentido había que añadir o omitir un punto de una letra, por ejemplo «aliombras-«alfombras); Errores de inclusión o supresión de una letra (L-A) (por ejem. abolque»-«bloque»).

b) Errores para cambiar el sentido de la palabra, subdivididos de nuevo en tres: de rotación (R-C) (como antes, hay que rotar una letra para que la palabra pase de un significado a otro, v. gr., «dijo» «dejo»); de omisión o añadido de un punto (OA-C) (v. gr. «pana»-«pata»); y de cambio de una letra (L-C) (v. gr. «caretas-«carretas).

3. Lectura de textos: Para esta parte de la investigación se trabajó con dos textos diferentes siempre leídos de manera táctil independientemente del grado de resto visual que tuvieran los sujetos:

a) Un texto largo escrito en tres hojas y con un nivel sintáctico y semántico bastante elemental:

La primera hoja era de entrenamiento; en la segunda se pretendía medir la velocidad de lectura en un texto normal y las estrategias de movimientos de las manos y en la última hoja se incluyeron palabras con las erratas que se ha- 
bían manipulado en la. lista de palabras ya comentada; es decir errores que se construían teniendo en cuenta todas las posibilidades de interacción dos a dos de las variables tipo de error inducido (rotación, omisión o añadido, y cambio de una letra) y efecto en el significado (añadir, quitar, cambiar), siempre teniendo en cuenta el contexto de la historia que se contaba.

Se utilizaron dos cuentos diferentes con esta misma estructura y con un nivel de dificultad equivalentes, uno para ser leído en voz alta y otro para hacerlo en silencio. En este segundo caso el sujeto recibió la consigna siguientes: «Vas a leer un texto. Después te haremos algunas preguntas sobre lo que dicè. Estas preguntas constaban de 20 cuestiones de comprensión sobre el texto que acaban de leer, 10 de ellas referidas a las dos primeras hojas y las 10 últimas al texto con erratas intencionadas, cada una de estas preguntas hacía referencia a la parte en donde estuviesen dichas erratas. El orden de presentación de los textos se contrabalanceaba.

b) El último texto usado no tenía sentido, era un texto con unidad sintáctica pero no semántica. Como en el caso anterior se presentaron dos textos de este tipo de estructura similar, uno para lectura silenciosa y otro para lectura en voz alta.

Las lecturas de los sujetos fueron grabadas mediante una cámara de video colocada verticalmente. Un espejo situado en la parte superior del texto con una inclinación de 45 grados con respecto al plano horizontal permitía apreciar los movimientos de presión de las manos al leer el texto.

Se tomaron datos sobre tiempos, recorridos, errores y nivel de comprensión para cada sujeto de cada grupo y con cada tipo de texto.

Por último se realizó un dictado muy breve de un texto de nivel sintáctico y semántico elemental, con la finalidad de comparar los errores producidos con: los que se daban en el casoo de la lectura.

\section{Sujetos}

Ciegos totales de nacimiento y ambliopes, todos ellos sin deficiencias asociadas. Se eligieron sujetos con diversas edades y diferentes niveles de destreza lectora. La muestra estudiada fue dividida en cinco grupos cada uno de ellos con 4 ciegos totales y 4 amblíopes, la variabilidad de edades en cada nivel escolar nunca era superior a los 2 años. El primer grupo estaba formado por sujetos del ciclo inicial de la EGB; el segundo nivel eran sujetos del ciclo medio de EGB; 3. sujetos del ciclo superior de EGB; 4. estudiantes de BUP; 5 . adultos, profesores de los colegios de la ONCE.

\section{Pruebas estadísticas}

Para la comparación de los datos entre las diferentes modalidades de las V. Is. manipuladas se realizó un ANOVA con inclusión de medidas repetidas, ẹn aquellas interacciones significativas con un nivel de confianza del $95 \%$ se utilizó para las comparaciones dos a dos entre las diferentes modalidades la prueba de Tukey con un $\alpha=0.5$.

El procedimiento de análisis de los resultados que se siguió para el estudio del movimiento de las manos, fue el de una observación estructurada, con categorías de observación cerradas y exhaustivas, por medio de varios juećes entrenados a tal efecto. 


\section{RESULTADOS}

Para resumir y englobar los múltiples resultados y los numerosos análisis que hemos realizado a lo largo de este trabajo seguiremos el siguiente orden. Primero extractaremos las conclusiones sobre los tiempos de lectura en los cuatro grandes bloques de pruebas, letras, palabras, textos y escritura. Después compararemos los tipos de errores que se han producido entre esos cuatro bloques de tareas. Para finalmente resumir las conclusiones referidas a los movimientos de las manos durante la lectura.

\section{Comparaciones de Tiempos de Lectura entre Lista de Letras, Palabras, Textos y Escritura.}

En resumen, en cada bloque de pruebas los tiempos que se ha tardado en realizar cada tarea (ver tabla I) se distribuyen de la siguiente forma:

En la lista de letras, conforme aumentaba la edad disminuía el tiempo en que se tardaba en leer.

En la lista de palabras, ocurría prácticamente lo mismo. Las diferencias significativas se encuentran entre el nivel I y todos los demás y entre los niveles de escolarización segundo y tercero con respecto al cuarto y al quinto. Se han encontrado ciertas diferencias entre ciegos y amblíopes en los tres primeros niveles de edad, en el sentido de que tardan más en leer los amblíopes de esos niveles que los ciegos.

En los textos, vuelve a observarse un fenómeno semejante. La velocidad es inversamente proporcional al nivel escolar, siendo en los tres últimos niveles más próximas. Se ha manifestado cierta tendencia a que los amblíopes tarden más en leer que los ciegos, sobre todo en aquellos textos con erratas intencionadas.

En la prueba de escritura (el dictado) disminuye también el tiempo de escritura conforme aumenta la edad.

En todas las tareas, como hemos visto, el tiempo disminuye conforme se incrementa la edad, no encontrándose diferencias ya entre los dos últimos niveles. Estos resultados concuerdan con los datos de otros estudios (Nolan y Kederis, 1969). Nos encontramos, pues, con que hasta que no finaliza la EGB los sujetos ciegos escolarizados siguen haciendo progresos notables en su aprendizaje de la lectura y la escritura.

Solamente en la lectura de textos en los tres primeros niveles de edad y sobre todo en aquellas tareas en las que se incluía errores intencionales, se han puesto de manifiesto cierto retraso de los amblíopes con respecto a los ciegos. A los amblíopes se les obligaba a leer los textos en braille de manera táctil y no visualmente, tal y como estaban acostumbrados casi todos. Nuestros datos demuestran que los amblíopes son más torpes cuando leen táctilmente que los ciegos totales, por su preferencia a una lectura visual del braille. Además los amblíopes (quizá debido a la falta de práctica con la lectura exclusivamente táctil) se muestran menos confiados ante la lectura de textos «raros», donde se les incluyen erratas que no esperaban, que los niños ciegos de la misma edad, con un poco más de destreza en la lectura táctil.

En nuestro estudio encontramos unas velocidades de lectura muy semejantes a las de trabajos anteriores. Así, los niños de primer ciclo de EGB alcanzan una media de $33 \mathrm{p} / \mathrm{m}$ en la lectura de un cuento, los del ciclo superior de la EGB llegan a las $80 \mathrm{p} / \mathrm{m}$; y los adultos obtienen una media de $121 \mathrm{p} / \mathrm{m}$. Es de destacar que las personas que tenían algún resto visual obtenían unos resultados inferiores en algo más de la mitad que los de sus compañeros ciegos totales. En 
algunos casos se les permitió después de pasada la prueba que utilizasen su resto visual para la lectura, entonces sus medias no sólo se igualaban, sino que incluso llegaban a ser algo superiores a las de los ciegos sin resto visual.

TABLA 1

Velocidad de lectura, medias de palabras (letras, en el caso de la primera lista) por minuto en las diferentes tareas estudiadas

\begin{tabular}{|l|c|c|c|c|}
\hline NIVEL DE EDAD & LETRAS & PALABRAS & TEXTOS & DICTADOS \\
\hline I (1 ciclo EGB) & 18,51 & 7,48 & 25,48 & 1,50 \\
\hline II (2 ciclo EGB) & 21,58 & 14,65 & 40,49 & 2,80 \\
\hline III (3 ciclo EGB & 35,28 & 20,55 & 55,57 & 26,60 \\
\hline IV (BUP) & 33,33 & 30,50 & 81,05 & 33,70 \\
\hline V (adultos) & 57,70 & 36,67 & 93,11 & 17,10 \\
\hline
\end{tabular}

\section{Comparaciones de los Tipos de Errores entre Tareas}

Como sabemos, el braille es un sistema que se basa en las localizaciones espaciales de puntos, algo que no ocurre con el sistema de lecto-escritura de los videntes. Es por ello lógico que se produzcan confusiones explicables principalmente por las características de este código de lecto-escritura. Los resultados que hemos obtenido parecen que van en ese sentido (ver tablas II y III). Así, en la lista de letras los errores más comunes han sido los de rotaciones de formas, y en la lista de palabras los errores de omisión o añadido de puntos y de rotación han sido tan importantes, cada uno de ellos, como los de cambio o introducción de letras $(\mathrm{L})$, en los cuales no hay influencia aparente del sistema de lecto-escritura braille. En los diferentes textos y en el dictado aunque estos errores «L» fueron más numerosos que los específicos del braille, la importancia de estos últimos no disminuía e incluso, en los estudiantes de BUP y los adultos, eran tan importantes en número como los primeros. De nuestro diseño y del análisis de los resultados podemos aventurar una explicación sobre el origen y las características más específicas de esos tipos de errores.

TABLA II

Porcentaje de los errores cometidos en las listas de letras y palabras

\begin{tabular}{|l|c|c|}
\hline & LETRAS & PALABRAS \\
\hline Rotación & $70 \%$ & $33,9 \%$ \\
\hline Omisión-añadido & $20 \%$ & $34,4 \%$ \\
\hline Orientación & $9 \%$ & - \\
\hline Cambio de una letra & - & $31,7 \%$ \\
\hline
\end{tabular}

Así, los errores de rotación parecen deberse a problemas espaciales de reconocimiento de formas, entendiendo por «forma» en este caso la especial configuración de celdillas que constituye cada letra en braille. Si esto fuese así, estos errores aparecerían más frecuentemente cuando la tarea estuviera enfocada hacia dicho reconocimiento, $y$, en consecuencia serían menos frecuentes cuanto 
TABLA III

Porcentaje de los errores cometidos en los textos, segün el nivel de edad

\begin{tabular}{|l|c|c|c|c|c|}
\hline & EGB-1 & EGB-2 & EGB-3 & BUP & ADULTOS \\
\hline Rotación & $18,6 \%$ & $54,7 \%$ & $20,4 \%$ & $24,1 \%$ & $42,1 \%$ \\
\hline Omisión-añadido & $21,2 \%$ & $13,5 \%$ & $22,4 \%$ & $29,1 \%$ & $35,8 \%$ \\
\hline Cambio de letra & $59,7 \%$ & $31,8 \%$ & $57,2 \%$ & $46,8 \%$ & $22,1 \%$ \\
\hline Media total de errores & 8,06 & 6,86 & 3,48 & 2,2 & 4,16 \\
\hline
\end{tabular}

mayor fuera la interferencia de reglas gramaticales y contextuales. Así es como ha ocurrido. Las confusiones por rotación han sido más numerosas en la lista de letras (que exigía sólo un reconocimiento de signos), seguida de la lista de palabras (donde intervenía la configuración ortográfica), y disminuyendo aún más en los diferentes textos (donde hay influencia tanto ortográfica como contextual). En el dictado ha sido donde menos erratas de este tipo se han producido debido, quizás, a que en el proceso de la escritura no existe percepción táctil de la forma, sino sólo traslación de una configuración imaginada o aprendida a un papel por medio de una pauta o de una máquina.

El error de omitir o añadir un punto en la celdilla es un error más amecánico», es un error en la percepción táctil que hace que no se aprecie un punto determinado de una letra para fusionarlo con la siguiente, o al contrario. Estos errores, por tanto, se producirían más cuando aparezcan varias letras juntas y, ocurriría con mayor frecuencia si no existe influencia del contexto (como es el caso de la lista de palabras), pues dicho contexto permitiría anticipar el sentido de la palabra, haciendo entonces menos necesaria la percepción detenida de cada letra (caso de los textos y del dictado). Cabe esperar, pues que donde menos aparezca este tipo de errores sea en la tarea en la que las letras se encuentren aisladas (lista de letras). Nuestros resultados concuerdan exactamente con esta predicción.

Las confusiones de cambiar o introducir una letra o letras en la palabra (L) se producen cuando existe un contexto fuerte que permite hacer un cambio tan «grosero» como éste sin que cambie el sentido general de lo escrito, y cuando, por ese contexto se puede anticipar qué es lo que vendrá a continuación en el proceso de la lectura, haciendo entonces menos importante el papel de la percepción letra a letra en cada palabra. Es por ello que donde más han aparecido dichas confusiones han sido en los textos y en el dictado, seguido de la lista de palabras, donde el contexto es mucho más débil y la posibilidades de anticipar más reducida. En la lista de letras lógicamente no podía aparecer este tipo de error.

En cuanto a la otra variable que hemos analizado en algunas tareas, el «Efecto del significado», cabe resaltar que se ha producido lo esperado pero con algunos matices (ver tabla IV). Es decir, las confusiones que se han dado han sido en su mayoría para dar sentido a la palabra, y en su caso al texto. Sobre todo, como se presuponía, ha ocurrido este hecho en los grupos de edad superiores, donde la lectura es más comprensiva, lo que obliga a que se tienda más a dar sentido a una palabra que no lo tiene. En algunos casos también aparecían con cierta abundancia ciertos tipos de respuestas que no se esperaban (como la cantidad de errores «neutros» en la lista de palabras), aunque se produjo principalmente en los grupos de menor edad donde la estructura de la tarea, quizá en 
algunos casos demasiado «artificial» (lista de palabras), puede. haber facilitado la aparición de tales errores.

\section{TABLA IV}

Medias correspondientes a los números de errores cometidos teniendo en cuenta la edad $y$ el efecto del significado, en la lectura de los textos

\begin{tabular}{|l|c|c|c|c|c|}
\hline & EGB-1 & EGB-2 & EGB-3 & BUP & ADULTOS \\
\hline Añade & 1,50 & 1,83 & 1,42 & 1,42 & 3,10 \\
\hline Cambia & $2,66$. & 1,62 & 1,04 & 0,42 & 0,71 \\
\hline Quita & 6,41 & 2,37 & 2,04 & 0,99 & 0,33 \\
\hline Neutro & 0,50 & 0,66 & 0,14 & 0,09 & 0,09 \\
\hline
\end{tabular}

Por otro lado, y recogiendo lo dicho un poco más arriba podemos afirmar que el número de errores en la lectura braille es menor cuando más facilidad exista para repasar, para tener una retroalimentación adecuada de lo que se lee o escribe, y cuanto más posibilidad haya de anticipar, de poder prever lo que puede venir a continuación de lo escrito. Por eso, se cometen más errores en la escritura que en la lectura, porque cuando se escribe, sobre todo con la pauta, es menos posible repasar, tener una información adecuada de qué es lo que se acaba de poner. También por esta misma razón en los textos sin sentido, en donde no hay estructura semántica y obviamente tampoco se puede anticipar lo que vendrá, se comete más errores que en el dictado donde, a su vez, como ya hemos dicho, son mayores estas confusiones que en la lectura normal.

Utilizamos en nuestro trabajo unas categorías de errores parecidas a las usadas por Uniform Type Comittee (1913), Ashcroft (1960) y Nolan y Kederis (1969). En estas investigaciones todos los errores disminuían más o menos homogéneamente con la edad. Según nuestros datos, la importancia relativa de los distintos tipos de equivocaciones consideradas variaba de acuerdo con el tipo de tarea y no sólo con la edad. Así, los errores específicos del braille (rotaciones y omisiones-añadidos de puntos) disminuían con la edad, pero tal disminución se produjo en menor magnitud que las confusiones comunes con la lectura visual, este hecho fue más claro en la tarea de lectura de un texto. Esta mayor estabilidad de los errores propios del braille obliga a que se les deba prestar una mayor atención en la enseñanza de tal sistema. Aunque los errores de omisión-añadido de puntos en ninguna tarea fueron más importantes que los demás, tanto este tipo de confusiones como los errores por rotación se producían más en palabras con configuraciones complejas (aquéllas que incluían los puntos inferiores 3 y 6), tal y como ocurrió en los trabajos antes mencionados.

El hecho de que en nuestro estudio los errores de omisión y añadido no sean tan importantes como en los trabajos que le preceden puede deberse a que nuestra categorización de errores consideraba como confusiones de este tipo sólo a las omisiones o añadido de un único punto. Incluimos las introducciones o las supresiones de más de un punto de la celdilla braille dentro de la categoría que hemos llamado «L (cambio de una letra). El omitir o añadir más de un punto determina un cambio tan grosero, son tantas las posibilidades de letras distintas que se pueden formar de esta manera, que creemos difícil que estos errores se deban exclusivamente a la forma táctil de recoger la información. Por otra parte si admitiéramos la adición y/o supresión de más de un punto, caerían 
dentro de este apartado muchos de los errores que hemos clasificado dentro de otros tipos, con lo que el análisis de errores creemos que disminuiría en precisión.

Pero además nuestro trabajo ha encontrado otros factores que pueden explicar la causa de estas equivocaciones. Así, los dos tipos de confusiones típicas del braille se han producido en letras de poco uso en español, las letras que incluyen el punto 6 son las últimas del abecedario, en su mayoría poco utilizadas en nuestra lengua. Por otro lado, los errores de rotación se han observado además, en letras que tenían un eje dominante oblicuo en su configuración global. A esto hay que añadir que las vocales acentuadas en braille son totalmente distintas a esas vocales sin acentuar, constituyéndose con la inclusión de los puntos inferiores y formando una configuración oblicua, lo que las hace, como ponen de manifiesto nuestros datos, especialmenté difíciles de reconocer. Por otro lado parece que el sentido del texto también influye a la hora de cometer estos tres tipos de errores, la magnitud de éstos disminuía conforme aumentaba la influencia del contexto.

El fuerte efecto de la familiaridad encontrado por Nolan y Kederis (1969), en el sentido que en aquellas palabras menos conocidas por el sujeto se tardaba más en su lectura y se cometían un número de errores notablemente mayor que en aquellas palabras más usadas, se da también en nuestro trabajo. Incluimos unas 6 palabras muy poco conocidas por los sujetos en el texto, que fueron pronunciadas de 38 maneras diferentes generando 42 errores distintos. Estas confusiones se distribuyen en general entre los tres tipos de errores de la misma forma que lo hace el texto entero.

\section{EL. MOVIMIENTO DE LAS MANOS EN LA LECTURA}

Del análisis descriptivo de los movimientos que se producen en la lectura de textos, se pueden deducir las siguientes conclusiones.

En primer lugar la mayor parte de los sujetos utilizan las dos manos para leer, de cada mano sólo emplean el dedo índice, aunque sí se notan diferencias en cuanto a la destreza, siendo los sujetos más pequeños los menos diestros y encontrándose más variabilidad en cuanto al uso de una o dos manos, también en estos grupos.

El movimiento general de lectura no parece que varíe escalonada y exclusivamente por el nivel de edad y el tipo de deficiencia visual. Nuestros datos muestran un cierto cambio en los patrones de movimientos de las manos que no se corresponden estrictamente con la división por edades que hemos hecho, aunque la dirección del cambio está bien definida. La tendencia de dicha evolución va desde la lectura unimanual en los sujetos más pequeños (tipo I de Kusajima, 1974), hasta una lectura bimanual disjunta en los últimos niveles de edad (Bertelson, Mousty y D'Alimonte, 1985; tipo IV de Kusajima), pasando por el patrón segundo de Kusajima, en el que los dos dedos permanecen juntos todo el tiempo, es decir en donde no existen trayectos unimanuales, este tipo se ha encontrado sólo en el primer y en el segundo nivel de edad; por último el tercer patrón, en el que se inician ya ciertos movimientos de exploración disjunta, se da en todos los grupos de EGB y en algunos sujetos de los otros niveles superiores.

Por lo que se refiere al modo en que se produce el cambio de una línea a la siguiente, hemos encontrado diferencias evolutivas entre los sujetos. Los ni- 
ños más pequeños (primer nivel de edad, ciegos y amblíopes), que son los que menos nivel de instrucción lectora tienen, cambian de renglón con las dos manos juntas de forma que ambas retroceden por el mismo renglón que acaban de leer. En cambio en el segundo nivel de edad, las manos siguen yendo juntas hasta casi el final de la línea, momento en que el dedo izquierdo baja, inmediatamente antes que el derecho, al renglón siguiente y retrocede por éste hasta el principio. En los restantes niveles de edad las manos se separan aproximadamente al llegar a la segunda mitad de la línea. El dedo izquierdo se coloca rápidamente al principio del renglón siguiente mientras el derecho termina de leer la línea, momento en que el dedo izquierdo empieza a leer el renglón nuevo. Es decir, parece que conforme se avanza en el nivel de instrucción lectora los dedos cobran una mayor autonomía, como si no necesitasen tanto tiempo del apoyo y referencia del otro dedo.

La coordinación dicción-movimientos no parece diferir de la de los videntes, en el sentido de que los sujetos más pequeños leen principalmente de forma silábica, mientras que los más mayores lo hacen palabra por palabra o por unidades semánticas.

\section{CONCLUSIONES E IMPLICACIONES EDUCATIVAS}

Los errores de rotación y en menor medida los de omisión y añadido, como ya conocemos, han sido importantes en las tareas estudiadas. Este hecho se puede explicar por la característica espacial de esta lectura, pero si tenemos en cuenta que estas confusiones se producen, en su mayoría, en los dos primeros niveles de edad, no podemos descartar la influencia de otro factor, el sistema de escritura usado por estos niños. Los niños de primer ciclo de EGB de los colegios de la ONCE donde recogimos los datos, escribían todos con «pauta». Como ya vimos en la introducción, con este procedimiento las letras debían escribirse rotadas 180 grados en relación a como se lee, lo que puede hacer pensar, según los resultados que tenemos, que puede inducir a confusiones en la lectura de estos niños. En estos mismos niveles, además, el número de errores en la tarea de escritura ha sido significativamente mayor que en la de lectura, quizá debido, como ya explicamos, a la dificultad que tienen de repasr lo escrito. Podría ser conveniente, en consecuencia, que se reconsiderase más detenidamente a estas edades el aprendizaje de su sistema de escritura.

Dentro de estos mismos errores de rotación las confusiones se han agrupado en las letras complejas (que usaban los puntos 3 y/o 6) y con un eje dominante oblicuo, lo cual era esperable de antemano, al ser estas palabras las que más confusiones de formas pueden generar. Tres grupos de letras de similar configuración han originado estos errores, la d-f-j-h, la n-z-è, la e-i y la à-ú; ò-u. En la enseñanza del braille podrían prestarse atención a estos resultados. El caso de las vocales acentuadas es singular en este sistema, cada vocal acentuada no se parece en modo alguno a esa vocal sin acentuar (excepto la $i-i)$, lo que no ocurre en la lectura para videntes, esto implica que para el mismo fonema los niños ciegos hayan de aprender dos signos distintos, uno de los cuales (el acentuado) es especialmente complejo; todo ello puede generar problemas que no se dan con los niños videntes.

En el trabajo de Newman, Hall y colaboradores (1982) se intentó estudiar la influencia que tenía en la lectura braille el aprendizaje de este sistema de forma visual o háptica, y el efecto del tamaño de la celdilla. Los resultados de- 
mostraron que las condiciones de aprendizaje visual eran significativamente más rápidas y más óptimas que el aprender braille hápticamente, incluso en el caso en que se aprenda visualmente y se lea luego táctilmente. En nuestro trabajo, como ya hemos visto, el hecho de tener cierto resto de visión obligaba a un mayor esfuerzo a la hora de leer, aunque no determinaba ni un número, ni unos tipos de confusiones muy diferentes a las de sus compañeros ciegos totales. Con estos datos queremos recoger la recomendación de Newman et al., de que dado que la visión puede significar una ventaja para el aprendizaje del Braille, las personas con algún resto visual deberían de aprender visualmente este sistema siempre que sea posible. En este mismo trabajo se encontró que cuando se leía con unas celdillas de un tamaño más grande de lo normal las diferencias entre aprendizaje táctil y visual no eran tan grandes. Por lo que parece recomendable también que la enseñanza háptica del braille se inicie con celdillas más grandes.

Para mejorar la instrucción en este sistema de lecto-escritura no basta con tener en cuenta estas recomendaciones, es conveniente hacer un análisis interno, lingüístico y ortográfico, del sistema braille (Hamp y Caton, 1984). Tal análisis debe de tener en cuenta los conocimientos que sobre los términos lingüísticos tienen los niños, sobre todo si se trata de la enseñanza de un sistema Braille con abundantes contracciones y simplificaciones como el braille 2, en el caso de la lengua inglesa, y la estenografía en el castellano.

Un aspecto en el que merece la pena detenerse es en el uso efectivo que los ciegos hacen de esta modalidad de acceso a la información escrita. Ni que decir tiene que el braille es el sistema de escritura extensivamente utilizado durante el período de escolarización. Pero, ¿cuál es el uso que de él hacen una vez que abandonan la escuela? El trabajo de Mack (1984), basado en una encuesta a ciegos adultos norteamericanos, pone de manifiesto el escaso uso que estos sujetos hacen del braille, que queda prácticamente restringido a cuestiones como tomar notas breves, apuntar números de teléfonos, etc., mientras que el grueso de la información escrita a la que accedían lo hacían a través de grabaciones magnetofónicas o por personas que les leían. Es de destacar que un gran número de ellas no leían por placer y que cuando lo hacían por motivos de trabajo utilizaban el optacón. Por lo que se refiere a la escritura la mayoría optaban por el uso de la máquina de escribir normal o por la utilización de cintas magnetofónicas. De todos modos, hay que señalar que todos los sujetos subrayaban que el braille les resultaba insustituible por la autonomía que les permitía, así como por algunas ventajas inherentes al uso de la información escrita, entre las cuales está la disposición espacial de las frases en la hoja de papel. En los últimos años se han desarrollado una serie de aparatos al amparo del auge de la microinformática, que pueden considerarse como un complemento del sistema braille. Se pueden encontrar en el mercado aparatos especialmente diseñados para invidentes tales como: ordenadores con voz, como el «Microbraille MB-2400», y el $«$ Small Talk»; lectores de caracteres ópticos, como el «Dest $113 \mathrm{~A} »$; editores braille, como el «VersaBraille Il $»$; impresoras braille, como el «Braille ' $N$ ' Print»; sintetizadores de voz, etc. En algunas instituciones se están creando programas educativos muy elaborados para la enseñanza de las habilidades necesarias para el mejor uso de dichas aplicaciones (Koening, Mack, et al., 1985).

Otro aspecto que conviene tener en cuenta es la comparación entre el braille y otras formas de acceder a la información literaria. El trabajo de Tuttle (1974) trata precisamente de comparar las ventajas relativas que pueden existir entre tres formas distintas de acceso a la lectura por parte de los ciegos: el braille y 
la grabación magnetofónica a velocidad normal y a velocidad aumentada. Su muestra estaba constituida por un grupo de 104 ciegos entre 14 y 21 años que «leyeron» un texto equivalente en cada una de estas tres modalidades. Sus conclușiones indican que no hay una diferencia global en nivel de comprensión entre las tres condiciones experimentales, mientras que la lectura braille se hacía a mitad de la velocidad que la «lectura» en cinta a velocidad normal y llevaba tres veces más tiempo que la «lectura» mediante cinta a velocidad aumentada. De todos modos, estê autor señala que existe una gran dispersión de resultados, algunos sujetos comprendían mejor con un sistema y otros con uno diferente, por lo que, a su juicio, no parece recomendable la opción exclusiva por uno de ellos. Como dato interesante hay que señalar que, según los datos de Tuttle, la velocidad óptima de alecturas mediante cinta a velocidad comprimida para el sujeto medio es de 275 palabras por minuto, lo que ya es una velocidad de lectura muy importante para un ciego.

A los datos que acabamos de presentar hay que añadir otros resultados de interés encontrados por algunos autores y que van en la línea de lo que acabamos de exponer. Mientras que Hartlage (1963) no encontró diferencias significativas entre ciegos y videntes respecto a la comprensión auditiva a velocidad normal, Foulke (1964) encontró que los ciegos comprenden el habla comprimida mejor que los videntes, lo que puede achacarse a su mayor entrenamiento en «leer» escuchando. Tanto para el mejor uso de esta última técnica como para la utilización óptima de las posibilidades acústicas de los ordenadores, sería interesante el estudio y entrenamiento de las habilidades de escucha y audición implicadas.

Para concluir, quisiéramos resaltar un hecho importante. Nuestros datos tanto en velocidad de lectura, como en número y tipos de errores, como en el movimiento de las manos, no se estabilizan hasta los últimos niveles de edad, es decir, a partir de BUP. Este desarrollo es diferente, algo más retrasado, al que se observa en la lecto-escritura de los videntes. Nolan y Kederis señalan cómo hasta al final del wunior-School» (equivalente a nuestra EGB) siguen apareciendo avances en la lectura y escritura braille, en contraste con lo que ocurre con los videntes. Este hecho lo achacan estos autores a cierto retraso madurativo por parte de los invidentes. Es importante hacer notar que en estudios anteriores (Ochaita, 1984; Rosa, 1981; Rosa et al., 1986) se muestra cómo los ciegos manifiestan ciertos retrasos en la realización de tareas manipulativo-figurativoespaciales que miden desarrollo cognitivo. Dicho retraso desaparece en las mismas edades que lo hace en la lectura. Esto sugiere que podría haber una conexión entre el desarrollo cognitivo y las habilidades de lecto-escritura. Sería objeto de otras investigaciones el tratar de explicar si tal relación existe y cuál es su naturaleza. 